\title{
Cultura catalana spazio europeo
}

\author{
Catalan culture, European space
}

\author{
Nancy De Benedetto \\ alfonsina.debenedetto@uniba.it
}

Università di Bari Aldo Moro

Meno di un anno fa, il 15 e il 16 novembre 2019, si è svolto all’Università di Bari «Aldo Moro» il convegno «Cultura catalana spazio europeo», organizzato dall'Associazione italiana di studi catalani. L'iniziativa si articolava su due mezze giornate di argomento diverso a cui rispondono le istanze su cui si apre la prospettiva, per qualche verso obbligata, del nostro mandato.

Da una parte era per noi imprescindibile perseguire l'attualità culturale della discussione sulla democrazia in Europa e le tematiche identitarie nazionali di cui la Catalogna è stata destabilizzante e forse incompresa protagonista negli ultimi anni, in un contesto europeo molto pervaso da inamovibile economicismo e ributtanti sovranismi. Ci sembra irrinunciabile seguire e contribuire correttamente alla questione che vive una fase di forzata normalizzazione, sancita proprio in quei giorni baresi dall'emissione di una discutibile sentenza ai politici catalani. Avevamo invitato ad esprimersi e a confrontarsi voci diverse che inquadrano la questione dal punto di vista culturale, del Diritto europeo comparato, del diritto processuale comparato, dei diritti umani presso la Corte Europea. I risultati del confronto sono stati molto interessanti e speriamo che dalle pagine della benemerita RISCAT (Rivista Italiana di Studi Catalani) che ci pubblicherà, si possa contribuire allo sviluppo della discussione ancora in atto.

D’altra parte abbiamo dato voce al nostro versante più tradizionale e consolidato, quello degli studi filologici e letterari, ma sempre dal punto di vista di un auspicato allargamento dei confini nazionali della ricezione e della condivisione dei libri e degli autori che da València a Napoli, a Roma, alle 
rotte adriatiche delle navi del grande mare comune, da sempre costituiscono un unico grande e risalente bacino culturale. Gli interventi che seguiranno, tracciano il profilo che tra continuità e rinnovamento punteggiano in parte il piccolo grande ambito degli studi di catalanistica in Italia che, come ricordato da Annamaria Annicchiarico, videro una precoce nascita proprio a Bari agli inizi della seconda parte del secolo scorso.

Su un intento di ricollocamento della trasmissione di fonti aquitane nella lirica trobadorica catalana si sviluppa lo studio ambizioso e ben documentato di Riccardo Viel, che da subito e proficuamente ha colto l'invito a unire gli sforzi dei settori della filologia romanza e dell'iberistica a Bari.

Nel solco degli studi lulliani di più attuale e consolidato corredo ridisegna invece Simone Sari la produzione dell'esclusivo «canone» del monumentale autore mariorchino che mette la letteratura, con il suo mercato editoriale, al servizio della divulgazione del proprio complesso sistema scientifico e sapienziale.

Non manchiamo di segnalare l'attualità della revisione del concetto di canone europeo che Pietro Cataldi propone, non solo qui, nella direzione di una comparatistica elevata a sistema in cui un posto preferenziale spetta alla letteratura catalana. E in questa prospettiva si inquadra la nuova spinta agli studi ausiasmarchiani prodotti recentemente in Italia, non ultima la miscellanea March: leggere, editare, tradurre nel tempo, a cura di Annamaria Compagna e Núria Puigdevall; una seconda, importante miscellanea, è quella che ha presentato a Bari Cèlia Nadal, che ha visto la luce alla fine del 2018 per le torinesi Edizioni dell'Orso (Ausiàs March e il canone europeo, Aldinucci, Nadal Pascual eds.). Un passo decisivo sulla ricollocazione di March in Italia, non possiamo non accennarvi, è la traduzione che porta finalmente il poeta di Gandia in una tra le migliori sedi editoriali, cosa che, come nel 2013 per il Tirante il Bianco, ne segna una avvenuta accoglienza «istituzionale», al di fuori dei circuiti specialistici e della formazione accademica. Un Male strano. Poesie d'amore (Torino, Einaudi 2020), è una scelta di diciannove poemi che i curatori, Cataldi e Nadal, hanno tratto dall'esteso canzoniere marchiano; alcuni erano già apparsi in italiano in eccellenti prove precedenti (Di Girolamo, 1998), altri sono inediti. Si tratta di un gruppo costituito da sei componimenti: IX Amor se dol, XXVII Sobresdolor, XXXIII Sens lo desig, XXXVIII Si bé mostrau, XLIX A mal estrany, che dà il titolo alla raccolta, XCVIII Per lo cami de mort.

La sfida di questa traduzione è stata portare March nel nuovo millennio con l'intento di un ritorno alla lettera ronchiosa e impervia dell'originale, dove le possibilità di distensione della trama si riducono al minimo. Il March ragionatore proposto da Cataldi e Nadal dà del tu a un'amata accidentale, muovendosi preferenzialmente sul terreno della desolata scissione dark tra terrore e desiderio e della tracotanza frustrata della descrizione di Amore. 